\title{
Studies of Biologically Active Heterocycles: Synthesis, Characterization and Antimicrobial Activity of Some 2,5-Disubstituted-1,3,4-0xadiazoles
}

\author{
G. Nagalakshmi \\ Department of Pharmaceutical Chemistry, The Erode College of Pharmacy, Erode-638112, Tamilnadu, India
}

\begin{abstract}
In the present study, several 2,5-disubstituted-1,3,4-oxadiazoles (3a-o) have been synthesized by the condensation of 4-hydroxybenzohydrazide (1) with various aromatic acids (2a-o) in presence of phosphorus oxychloride. The structures of the newly synthesized compounds have been established on the basis of elemental analysis, UV, IR and ${ }^{1} \mathrm{H}$ NMR spectral data. The synthesized compounds were screened for their in vitro growth inhibiting activity against different strains of bacteria and fungi viz., Staphylococcus aureus, Bacillus subtilis, Bacillus megaterium, Escherichia coli, Pseudomonas aeruginosa, Shigella dysenteriae, Candida albicans, Aspergillus niger and Aspergillus flavus and the results were compared with the standard antibiotics such as chloramphenicol $(50 \mu \mathrm{g} / \mathrm{ml})$ and Griseofulvin $(50 \mu \mathrm{g} / \mathrm{ml})$ using agar diffusion technique. Compounds $\mathbf{3 b}, \mathbf{3 e}, \mathbf{3 g}, \mathbf{3 h}, \mathbf{3} \mathbf{j}, \mathbf{3 m}$ and $\mathbf{3 n}$ exhibited strong antibacterial activity and compounds 3a, 3d, $\mathbf{3 g}, \mathbf{3}$ and $\mathbf{3 i}$ showed good antifungal activity.
\end{abstract}

Key words: 2,5-disubstituted-1,3,4-oxadiazoles, 4-hydroxybenzohydrazide, phosphorus oxychloride, antimicrobial evaluation, agar well diffusion method.

\section{INTRODUCTION}

1,3,4-Oxadiazole is a versatile lead molecule for designing potential bioactive agents. The 1,3,4oxadiazole derivatives have been found to exhibit diverse biological activities such as antimicrobial ${ }^{1}$, anti-HIV $^{1}$, antitubercular ${ }^{2}$, antimalarial ${ }^{3}$, analgesic ${ }^{4}$, anti-inflammatory $^{5}$, anticonvulsant ${ }^{6}$, hypoglycemic $^{7}$ and other biological properties such as genotoxic studies $^{8}$ and lipid peroxidation inhibitor. ${ }^{9}$

Aryl alkanoic acids provide one of the most fascinating class of compounds recognized for various pharmacological actions like antipyretic, analgesic and anti-inflammatory activity ${ }^{10}$, and are

Correspondence to: G. Nagalakshmi,

Tel: 09843307741.

E-mail: gn178@yahoo.com used extensively in the symptomatic treatment of rheumatic fever, arthritis ${ }^{11}$ (rheumatoid, osteo and Jaundice arthritis), myocardial infarctions and management of primary dysmenorrheal. ${ }^{12}$ The major side effects in the use of aryl alkanoic acids is their gastric irritation, which is partly due to the corrosive nature of carboxylic acid group present in them. In order to reduce or mask the side effects of carboxylic moiety we planned to synthesize different 2,5disubstituted-1,3,4-oxadiazoles (3a-o) via the condensation of 4-hydroxybenzohydrazide with various aromatic acids in presence of phosphorus oxychloride respectively in the hope of getting potent biodynamic agents and evaluate their antimicrobial activity. 


\section{MATERIALS AND METHODS}

General experimental procedure. Column chromatography was carried out by using Merck silica gel 60 . The solvents used for elution were petroleum ether and ethyl acetate $(4: 1 \% \mathrm{v} / \mathrm{v})$. The purity of all the compounds were checked by TLC on precoated Silica- $60 \mathrm{~F}_{254}$ plates (Merck Mumbai) using Iodine vapours and UV light as detecting agents with the help of mobile phase ethyl acetate: acetone (9:1). The $R_{f}$ value of the synthesized compounds were determined and the melting points of the synthesized compounds were recorded by open capillaries in a liquid paraffin bath and are uncorrected. The absorbance maxima $\left(\lambda_{\max }\right)$ of the synthesized compounds were determined on a Systronics UVVisible double beam spectrophotometer (2201) in methanol. While the IR spectra of the synthesized compounds were recorded on a Perkin Elmer Spectrum RX I, FTIR spectrophotometer using potassium bromide (anhydrous IR grade) pellets. ${ }^{1} \mathrm{H}$ NMR spectra were acquired on AMX-400, NMR spectrometer using DMSO- $\mathrm{d}_{6}$ as solvent and TMS as an internal standard (chemical shift in $\delta \mathrm{ppm}$ ). The structures of the newly synthesized compounds were assigned on the basis of elemental analysis and were recorded on a Carlo Erba 1108 Heraeus at Regional Sophisticated Instrumentation Centre, CDRI,
Lucknow. Carbon, hydrogen and nitrogen analyses were within $\pm 0.4 \%$ of the theoretical values. All the chemicals used were of synthetic and AR grade and was procured from Agros-Organics, USA, S.D. Fine Chem. Ltd., Mumbai and Merck, Mumbai, India.

Synthesis of 2,5-disubstituted-1,3,4oxadiazoles (3a-o). A mixture of different aromatic acid(s) $(0.01 \mathrm{~mol})$ with 4-hydroxybenzohydrazide $(1.5215 \mathrm{~g}, \quad 0.01 \mathrm{~mol})$ in phosphorus oxychloride $(15 \mathrm{ml})$ was refluxed over a steam bath for 5-6 $\mathrm{h}$. The progress of the reaction was monitored by TLC (on precoated silica $60 \mathrm{~F}_{254}$ plates Merck, Mumbai) using ethylacetate: acetone (9:1) as developing solvents. The reaction mixture was cooled and poured onto crushed ice $(\sim 200 \mathrm{~g})$ with continuous stirring. The solid mass separated was neutralized with sodium bicarbonate solution $(10 \% \mathrm{w} / \mathrm{v})$. The resulting solid thus obtained was collected by filtration, washed well with cold water, dried in vaccum and crystallized from absolute ethanol (95\%) and analyzed spectroscopically. Adopting the above procedure fifteen different 2,5-disubstituted-1,3,4-oxadiazoles (3a-o) were synthesized and their characterization data are presented in Table 1. Yield and melting point of the product(s) were determined and summarized in below.

Scheme 1. Synthetic route for the preparation of some 2,5-disubstituted-1,3,4-oxadiazoles (3a-o).

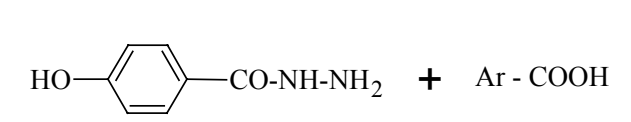

(1)

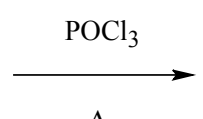

$\Delta$

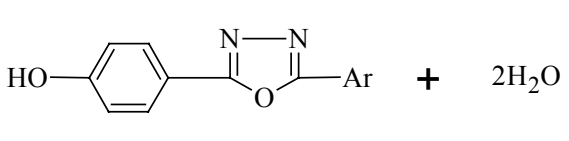

$(3 a-0)$

\begin{tabular}{|c|c|c|c|}
\hline Compound & Ar & Compound & Ar \\
\hline $3 a$ & $\mathrm{C}_{6} \mathrm{H}_{5}$ & $3 \mathbf{i}$ & $\mathrm{C}_{6} \mathrm{H}_{5} \mathrm{CONHC}_{6} \mathrm{H}_{4}$ \\
\hline 3b & $4-\mathrm{CH}_{3} \mathrm{C}_{6} \mathrm{H}_{4}$ & $3 \mathbf{j}$ & $4-\mathrm{OCH}_{3} \mathrm{C}_{6} \mathrm{H}_{4}$ \\
\hline $3 c$ & $\mathrm{CH}=\mathrm{CH}-\mathrm{C}_{6} \mathrm{H}_{5}$ & $3 \mathbf{k}$ & $3,4,5-\left(\mathrm{OCH}_{3}\right)_{3} \mathrm{C}_{6} \mathrm{H}_{2}$ \\
\hline 3d & $4-\mathrm{NH}_{2} \mathrm{C}_{6} \mathrm{H}_{4}$ & 31 & $\mathrm{C}_{5} \mathrm{H}_{4} \mathrm{~N}$ \\
\hline $3 \mathbf{e}$ & $4-\mathrm{NO}_{2} \mathrm{C}_{6} \mathrm{H}_{4}$ & $3 m$ & $2,4-(\mathrm{OH})_{2} \mathrm{C}_{6} \mathrm{H}_{3}$ \\
\hline $3 f$ & $3,5-\left(\mathrm{NO}_{2}\right)_{2} \mathrm{C}_{6} \mathrm{H}_{3}$ & $3 n$ & $3-\mathrm{NH}_{2} \mathrm{C}_{6} \mathrm{H}_{4}$ \\
\hline $3 g$ & $2,4-\left(\mathrm{NO}_{2}\right)_{2} \mathrm{C}_{6} \mathrm{H}_{3} \mathrm{NHC}_{6} \mathrm{H}_{4}$ & 3o & $2-\mathrm{OH} 3-\mathrm{CH}_{3} \mathrm{C}_{6} \mathrm{H}_{3}$ \\
\hline $3 \mathbf{h}$ & $2-\mathrm{NO}_{2} \mathrm{C}_{6} \mathrm{H}_{4} \mathrm{NHC}_{6} \mathrm{H}_{4}$ & & \\
\hline
\end{tabular}

4-(5-phenyl-1,3,4-oxadiazol-2-yl)phenol (3a). Yield: $72.41 \%(1.68 \mathrm{~g})$; $\mathrm{mp}: 212^{\circ} \mathrm{C}$; $\mathrm{R}_{\mathrm{f}}$ value: 0.762 ; UV $\left(\lambda_{\max }, \mathrm{nm}\right): 342.3$; IR $\left(\mathrm{KBr}, \mathrm{cm}^{-1}\right): 3601(\mathrm{O}-\mathrm{H}$ stretch), 3045 (aromatic C-H stretch), 1602, 1498,
1470 (aromatic $\mathrm{C}=\mathrm{C}$ ring stretch), $1634(\mathrm{C}=\mathrm{N}$ stretch), 1220 (C-O stretch), 1247 (asymmetric C-O$\mathrm{C}$ ring stretch), 1040 (symmetric C-O-C stretch), 1339, 1360 (in-plane O-H bend); ${ }^{1} \mathrm{H}$ NMR (DMSO- 
$\left.\mathrm{d}_{6}, \delta \mathrm{ppm}\right): 6.87-7.12(4 \mathrm{H}, \mathrm{m}$, aromatic protons), 7.27-7.41 (5H, m, aromatic protons), $5.57(1 \mathrm{H}, \mathrm{s}, \mathrm{Ar}-$ $\mathrm{OH})$.

\section{4-[5-(4-methylphenyl)-1,3,4-oxadiazol-2-}

yl]phenol (3b). Yield: $76.66 \%$ (1.98 g); mp: $220^{\circ} \mathrm{C}$; $\mathrm{R}_{\mathrm{f}}$ value: 0.851 ; $\mathrm{UV}\left(\lambda_{\max }, \mathrm{nm}\right): 270.2$; IR $\left(\mathrm{KBr}, \mathrm{cm}^{-}\right.$ $\left.{ }^{1}\right)$ : 3056 (aromatic C-H stretch), 3594 (O-H stretch), 1224 (C-O stretch), 1602, 1493, 1456 (aromatic $\mathrm{C}=\mathrm{C}$ ring stretch), 1247 (asymmetric $\mathrm{C}-\mathrm{O}-\mathrm{C}$ stretch), 1040 (symmetric C-O-C stretch), $1642(\mathrm{C}=\mathrm{N}$ stretch), 2960 (methyl C-H stretch yas $\mathrm{CH}_{3}$ ), 2872 (methyl C$\mathrm{H}$ stretch $\gamma \mathrm{s}^{\mathrm{CH}_{3}}$ ); ${ }^{1} \mathrm{H}$ NMR (DMSO-d ${ }_{6}, \delta \mathrm{ppm}$ ): 6.85-7.07 (4H, m, aromatic protons), 7.27-7.42 $(4 \mathrm{H}$, $\mathrm{m}$, aromatic protons), $2.39\left(3 \mathrm{H}, \mathrm{s}, \mathrm{Ar}-\mathrm{CH}_{3}\right), 5.59(1 \mathrm{H}$, $\mathrm{s}, \mathrm{Ar}-\mathrm{OH})$.

Table 1. Physical and analytical data of 2,5-disubstituted-1,3,4-oxadiazoles (3a-o).

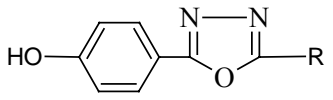

$(3 a-0)$

\begin{tabular}{|c|c|c|c|c|c|}
\hline \multirow{2}{*}{ Compound } & \multirow{2}{*}{$\mathbf{R}$} & \multirow{2}{*}{ Mol. for / Mol. wt } & \multicolumn{3}{|c|}{ Elemental analysis found (calcd)\% } \\
\hline & & & C & $\mathbf{H}$ & $\mathbf{N}$ \\
\hline \multirow[t]{2}{*}{$3 \mathbf{a}$} & $\mathrm{C}_{6} \mathrm{H}_{5}$ & $\mathrm{C}_{14} \mathrm{H}_{10} \mathrm{~N}_{2} \mathrm{O}_{2} / 238.24$ & 70.54 & 4.21 & $\overline{11.71}$ \\
\hline & & & (70.58 & 4.23 & 11.76) \\
\hline \multirow[t]{2}{*}{$3 \mathbf{b}$} & $4-\mathrm{CH}_{3} \mathrm{C}_{6} \mathrm{H}_{4}$ & $\mathrm{C}_{15} \mathrm{H}_{12} \mathrm{~N}_{2} \mathrm{O}_{2} / 252.26$ & 71.38 & 4.76 & 11.07 \\
\hline & & & (71.42 & 4.79 & 11.10) \\
\hline \multirow[t]{2}{*}{ 3c } & $\mathrm{CH}=\mathrm{CH}-\mathrm{C}_{6} \mathrm{H}_{5}$ & $\mathrm{C}_{16} \mathrm{H}_{12} \mathrm{~N}_{2} \mathrm{O}_{2} / 264.27$ & 72.68 & 4.54 & 10.55 \\
\hline & & & (72.72 & 4.58 & 10.60) \\
\hline \multirow[t]{2}{*}{ 3d } & 4- $\mathrm{NH}_{2} \mathrm{C}_{6} \mathrm{H}_{4}$ & $\mathrm{C}_{14} \mathrm{H}_{11} \mathrm{~N}_{3} \mathrm{O}_{2} / 253.25$ & 66.36 & 4.34 & 16.55 \\
\hline & & & $(66.40$ & 4.38 & 16.59) \\
\hline \multirow[t]{2}{*}{ 3e } & $4-\mathrm{NO}_{2} \mathrm{C}_{6} \mathrm{H}_{4}$ & $\mathrm{C}_{14} \mathrm{H}_{9} \mathrm{~N}_{3} \mathrm{O}_{4} / 283.24$ & 59.33 & 3.18 & 14.81 \\
\hline & & & (59.36 & 3.20 & 14.83) \\
\hline \multirow[t]{2}{*}{ 3f } & $3,5-\left(\mathrm{NO}_{2}\right)_{2} \mathrm{C}_{6} \mathrm{H}_{3}$ & $\mathrm{C}_{14} \mathrm{H}_{8} \mathrm{~N}_{4} \mathrm{O}_{6} / 328.23$ & 51.18 & 2.42 & 17.02 \\
\hline & & & (51.23 & 2.46 & 17.07) \\
\hline \multirow[t]{2}{*}{$3 g$} & $2,4-\left(\mathrm{NO}_{2}\right)_{2} \mathrm{C}_{6} \mathrm{H}_{3} \mathrm{NHC}_{6} \mathrm{H}_{4}$ & $\mathrm{C}_{20} \mathrm{H}_{13} \mathrm{~N}_{5} \mathrm{O}_{6} / 419.34$ & 57.25 & 3.10 & 16.67 \\
\hline & & & $(57.28$ & 3.12 & 16.70) \\
\hline \multirow{2}{*}{ 3h } & $2-\mathrm{NO}_{2} \mathrm{C}_{6} \mathrm{H}_{4} \mathrm{NHC}_{6} \mathrm{H}_{4}$ & $\mathrm{C}_{20} \mathrm{H}_{14} \mathrm{~N}_{4} \mathrm{O}_{4} / 374.34$ & 64.13 & 3.72 & 14.94 \\
\hline & & & $(64.17$ & 3.77 & 14.97) \\
\hline \multirow{2}{*}{$3 \mathbf{i}$} & $\mathrm{C}_{6} \mathrm{H}_{5} \mathrm{CONHC}_{6} \mathrm{H}_{4}$ & $\mathrm{C}_{21} \mathrm{H}_{15} \mathrm{~N}_{3} \mathrm{O}_{3} / 357.36$ & 70.57 & 4.21 & 11.72 \\
\hline & & & (70.58 & 4.23 & 11.76) \\
\hline \multirow{2}{*}{$3 \mathbf{j}$} & $4-\mathrm{OCH}_{3} \mathrm{C}_{6} \mathrm{H}_{4}$ & $\mathrm{C}_{15} \mathrm{H}_{12} \mathrm{~N}_{2} \mathrm{O}_{3} / 268.26$ & 67.13 & 4.48 & 10.42 \\
\hline & & & (67.16 & 4.51 & 10.44) \\
\hline \multirow{2}{*}{$3 \mathbf{k}$} & $3,4,5-\left(\mathrm{OCH}_{3}\right)_{3} \mathrm{C}_{6} \mathrm{H}_{2}$ & $\mathrm{C}_{17} \mathrm{H}_{16} \mathrm{~N}_{2} \mathrm{O}_{5} / 328.31$ & 62.16 & 4.89 & 8.51 \\
\hline & & & (62.19 & 4.91 & 8.53) \\
\hline \multirow{2}{*}{31} & $\mathrm{C}_{5} \mathrm{H}_{4} \mathrm{~N}$ & $\mathrm{C}_{13} \mathrm{H}_{9} \mathrm{~N}_{3} \mathrm{O}_{2} / 239.22$ & 65.24 & 3.78 & 17.54 \\
\hline & & & $(65.27$ & 3.79 & 17.56) \\
\hline \multirow{2}{*}{$3 \mathbf{m}$} & $2,4-(\mathrm{OH})_{2} \mathrm{C}_{6} \mathrm{H}_{3}$ & $\mathrm{C}_{14} \mathrm{H}_{10} \mathrm{~N}_{2} \mathrm{O}_{4} / 270.24$ & 62.20 & 3.72 & 10.35 \\
\hline & & & $(62.22$ & 3.73 & 10.37) \\
\hline \multirow{2}{*}{$3 n$} & $3-\mathrm{NH}_{2} \mathrm{C}_{6} \mathrm{H}_{4}$ & $\mathrm{C}_{14} \mathrm{H}_{11} \mathrm{~N}_{3} \mathrm{O}_{2} / 253.25$ & 66.37 & 4.36 & 16.56 \\
\hline & & & $(66.40$ & 4.38 & 16.59) \\
\hline \multirow[t]{2}{*}{30} & 2-OH3- $\mathrm{CH}_{3} \mathrm{C}_{6} \mathrm{H}_{3}$ & $\mathrm{C}_{15} \mathrm{H}_{12} \mathrm{~N}_{2} \mathrm{O}_{3} / \mathbf{2 6 8 . 2 6}$ & 67.12 & 4.50 & 10.42 \\
\hline & & & $(67.16$ & 4.51 & 10.44) \\
\hline
\end{tabular}

4-[5-(4-aminophenyl)-1,3,4-oxadiazol-2-

yl]phenol (3d). Yield: $84.89 \%$ (2.15 g); mp: $192^{\circ} \mathrm{C}$; $\mathrm{R}_{\mathrm{f}}$ value: 0.823 ; UV ( $\left.\lambda_{\max }, \mathrm{nm}\right): 263.6$; IR $\left(\mathrm{KBr}, \mathrm{cm}^{-}\right.$
4-\{5-[(E)-2-phenylvinyl]-1,3,4-oxadiazol-2-

yl\}phenol (3c). Yield: $79.46 \%(2.1 \mathrm{~g}) ; \mathrm{mp}: 242^{\circ} \mathrm{C} ; \mathrm{R}_{\mathrm{f}}$ value: 0.842 ; $\mathrm{UV}\left(\lambda_{\max }, \mathrm{nm}\right): 261.2 ; \mathrm{IR}\left(\mathrm{KBr}, \mathrm{cm}^{-1}\right)$ : 3606 (O-H stretch), 3042 (aromatic C-H stretch), 1220 (C-O stretch), 1609, 1493, 1466 (aromatic $\mathrm{C}=\mathrm{C}$ ring stretch), 1633 ( $\mathrm{C}=\mathrm{N}$ stretch), 3049 (alkene $\mathrm{C}-\mathrm{H}$ stretch), 1252 (asymmetric C-O-C ring stretch), 1045 (symmetric $\mathrm{C}-\mathrm{O}-\mathrm{C}$ stretch), $1642(\mathrm{C}=\mathrm{C}$ stretch, alkene), 1345, 1365 (in-plane O-H bend), 690 (outof-plane $\mathrm{C}=\mathrm{C}$ bend); ${ }^{1} \mathrm{H}$ NMR (DMSO- $\left.\mathrm{d}_{6}, \delta \mathrm{ppm}\right)$ : 6.87-7.07 $(4 \mathrm{H}, \mathrm{m}$, aromatic protons $), 7.22-7.48(5 \mathrm{H}$, $\mathrm{m}$, aromatic protons), $5.60(1 \mathrm{H}, \mathrm{s}, \mathrm{Ar}-\mathrm{OH}), 4.8-6.0$ $(2 \mathrm{H}, \mathrm{d}, \mathrm{CH}=\mathrm{CH})$. 
1040 (symmetric C-O-C stretch), 1644 (C=N stretch), 3520 (N-H stretch for aromatic primary amine, asymmetric), 3425 (N-H stretch, primary amine, symmetric), 1296 (aromatic C-N stretch for primary amine); ${ }^{1} \mathrm{H}$ NMR (DMSO-d $\left.{ }_{6}, \delta \mathrm{ppm}\right): 6.76-7.08(4 \mathrm{H}$, $\mathrm{m}$, aromatic protons), 7.23-7.52 $(4 \mathrm{H}, \mathrm{m}$, aromatic protons), $5.58(1 \mathrm{H}, \mathrm{s}, \mathrm{Ar}-\mathrm{OH}), 4.42\left(2 \mathrm{H}, \mathrm{s}, \mathrm{NH}_{2}\right)$.

\section{4-[5-(4-nitrophenyl)-1,3,4-oxadiazol-2-}

yl]phenol (3e). Yield: $74.14 \%(2.1 \mathrm{~g}) ; \mathrm{mp}: 228^{\circ} \mathrm{C} ; \mathrm{R}_{\mathrm{f}}$ value: 0.726 ; $\mathrm{UV}\left(\lambda_{\max }, \mathrm{nm}\right): 273.2 ; \mathrm{IR}\left(\mathrm{KBr}, \mathrm{cm}^{-1}\right)$ : 3052 (aromatic C-H stretch), 3588 (O-H stretch), 1226 (C-O stretch), 1602, 1495, 1470 (C=C stretch), 1250 (asymmetric $\mathrm{C}-\mathrm{O}-\mathrm{C}$ stretch), 1045 (symmetric C-O-C stretch), $1636 \quad(\mathrm{C}=\mathrm{N}$ stretch $), 1523$ (asymmetric $\mathrm{ArNO}_{2}, \mathrm{NO}_{2}$ stretch), 1347 (symmetric $\mathrm{ArNO}_{2}, \mathrm{NO}_{2}$ stretch), 852 (C-N stretch of $\mathrm{ArNO}_{2}$ ); ${ }^{1} \mathrm{H}$ NMR (DMSO-d $\left.6, \delta \mathrm{ppm}\right): 6.78-7.01 \quad(4 \mathrm{H}, \mathrm{m}$, aromatic protons), 7.27-7.62 $(4 \mathrm{H}, \mathrm{m}$, aromatic protons), $5.57(1 \mathrm{H}, \mathrm{s}, \mathrm{Ar}-\mathrm{OH})$.

\section{4-[5-(3,5-dinitrophenyl)-1,3,4-oxadiazol-2-} yl]phenol (3f). Yield: $73.78 \%$ (2.420 g); mp: $256^{\circ} \mathrm{C}$; $\mathrm{R}_{\mathrm{f}}$ value: 0.812 ; $\mathrm{UV}\left(\lambda_{\max }, \mathrm{nm}\right): 245.6$; $\mathrm{IR}\left(\mathrm{KBr}, \mathrm{cm}^{-}\right.$ $\left.{ }^{1}\right)$ : 3065 (aromatic C-H stretch), 3592 (Ar-OH stretch), 1224 (C-O stretch), 1602, 1493, 1455 (C=C ring stretch), 1256 (asymmetric $\mathrm{C}-\mathrm{O}-\mathrm{C}$ stretch), 1050 (symmetric $\mathrm{C}-\mathrm{O}-\mathrm{C}$ stretch), $1640(\mathrm{C}=\mathrm{N}$ stretch), 1542 (asymmetric $\mathrm{ArNO}_{2}, \mathrm{NO}_{2}$ stretch), 1358 (symmetric $\mathrm{ArNO}_{2}, \mathrm{NO}_{2}$ stretch), 858 (C-N stretch of $\mathrm{ArNO}_{2}$ ); ${ }^{1} \mathrm{H}$ NMR (DMSO-d $\mathrm{d}_{6}, \delta \mathrm{ppm}$ ): 6.87-7.05 $(4 \mathrm{H}, \mathrm{m}$, aromatic protons $), 7.28-7.48(3 \mathrm{H}, \mathrm{m}$, aromatic protons), $5.59(1 \mathrm{H}, \mathrm{s}, \mathrm{Ar}-\mathrm{OH})$.

\section{4-(5-\{4-[(2,4-dinitrophenyl)amino]phenyl\}-} 1,3,4-oxadiazol-2-yl)phenol (3g). Yield: 82.27\% (3.45 g); mp: $248^{\circ} \mathrm{C} ; \mathrm{R}_{\mathrm{f}}$ value: 0.762 ; $\mathrm{UV}\left(\lambda_{\max }, \mathrm{nm}\right)$ : 263.4; IR ( $\left.\mathrm{KBr}, \mathrm{cm}^{-1}\right)$ : 3030 (aromatic C-H stretch), 3595 (O-H stretch), 1226 (C-O stretch), 1596, 1492, $1458(\mathrm{C}=\mathrm{C}$ ring stretch), 1248 (asymmetric $\mathrm{C}-\mathrm{O}-\mathrm{C}$ stretch), 1047 (symmetric C-O-C stretch), 1647 (C=N stretch), 1525 (asymmetric $\mathrm{ArNO}_{2}, \mathrm{NO}_{2}$ stretch), 1342 (symmetric $\mathrm{ArNO}_{2}, \mathrm{NO}_{2}$ stretch), 854 (C-N stretch of $\left.\mathrm{ArNO}_{2}\right), 3325$ ( N-H stretch, aromatic secondary amine), 1298 (C-N stretch for secondary aromatic amine), 1358 (in-plane O-H bend); ${ }^{1} \mathrm{H}$ NMR $\left(\right.$ DMSO- $\left._{6}, \delta \mathrm{ppm}\right): 6.81-7.04(4 \mathrm{H}, \mathrm{m}$, aromatic protons), 7.18-7.61 (7H, m, aromatic protons), 5.58 $(1 \mathrm{H}, \mathrm{s}, \mathrm{Ar}-\mathrm{OH}), 2.18(1 \mathrm{H}, \mathrm{s}, \mathrm{NH})$.

4-(5-\{4-[(2-nitrophenyl)amino]phenyl\}-1,3,4oxadiazol-2-yl)phenol (3h). Yield: 75.86\% (2.84 g); mp: $218^{\circ} \mathrm{C}$; $\mathrm{R}_{\mathrm{f}}$ value: 0.824 ; UV ( $\left.\lambda_{\max }, \mathrm{nm}\right): 261.6$; IR $\left(\mathrm{KBr}, \mathrm{cm}^{-1}\right): 3070$ (aromatic C-H stretch), $3590(\mathrm{O}-\mathrm{H}$ stretch), 1230 (C-O stretch), 1601, 1495, 1474 (C=C ring stretch), 1242 (asymmetric C-O-C stretch), 1041 (symmetric C-O-C stretch), $1636(\mathrm{C}=\mathrm{N}$ stretch), 1538 (asymmetric $\mathrm{ArNO}_{2}, \mathrm{NO}_{2}$ stretch), 1350 (symmetric $\mathrm{ArNO}_{2}, \mathrm{NO}_{2}$ stretch), 852 (C-N stretch of $\left.\mathrm{ArNO}_{2}\right), 3330$ (N-H stretch, aromatic secondary amine), 1293 (C-N stretch for secondary aromatic amine), 1338, 1418 (in-plane O-H bend); ${ }^{1} \mathrm{H}$ NMR $\left(\right.$ DMSO-d $\left._{6}, \delta \mathrm{ppm}\right): 6.79-7.0(4 \mathrm{H}, \mathrm{m}$, aromatic protons), 7.21-7.48 (8H, m, aromatic protons), 5.59 $(1 \mathrm{H}, \mathrm{s}, \mathrm{Ar}-\mathrm{OH}), 2.21(1 \mathrm{H}, \mathrm{s}, \mathrm{NH})$.

\section{N-\{4-[5-(4-hydroxyphenyl)-1,3,4-oxadiazol-2-} yl]phenyl\}benzamide (3i). Yield: 81.15\% (2.9 g); $\mathrm{mp}: 250^{\circ} \mathrm{C}$; $\mathrm{R}_{\mathrm{f}}$ value: 0.782 ; $\mathrm{UV}\left(\lambda_{\max }, \mathrm{nm}\right): 268.6$; IR $\left(\mathrm{KBr}, \mathrm{cm}^{-1}\right)$ : 3048 (aromatic C-H stretch), $3589(\mathrm{O}-\mathrm{H}$ stretch), 1227 (C-O stretch), 1609, 1494, 1460 (C=C ring stretch), 1258 (asymmetric $\mathrm{C}-\mathrm{O}-\mathrm{C}$ stretch), 1049 (symmetric C-O-C stretch), $1645(\mathrm{C}=\mathrm{N}$ stretch), 3432 (N-H stretch, secondary amide), 1285 (aromatic C-N stretch for secondary amide), 1642 ( $\mathrm{C}=\mathrm{O}$ stretch, amide), 1346, 1420 (in-plane O-H bend); ${ }^{1} \mathrm{H}$ NMR $\left(\right.$ DMSO-d $\left._{6}, \delta \mathrm{ppm}\right): 6.87-7.07(4 \mathrm{H}, \mathrm{m}$, aromatic protons), 7.23-7.48 (9H, m, aromatic protons), 5.57 $(1 \mathrm{H}, \mathrm{s}, \mathrm{Ar}-\mathrm{OH}), 8.48(1 \mathrm{H}, \mathrm{s}, \mathrm{CONH})$.

4-[5-(4-methoxyphenyl)-1,3,4-oxadiazol-2-

yl]phenol (3j). Yield: $78.28 \%$ (2.1 g); mp: $232^{\circ} \mathrm{C} ; \mathrm{R}_{\mathrm{f}}$ value: 0.794 ; $\mathrm{UV}\left(\lambda_{\max }, \mathrm{nm}\right): 275.6$; $\mathrm{IR}\left(\mathrm{KBr}, \mathrm{cm}^{-1}\right)$ : 3048 (aromatic C-H stretch), 3604 (O-H stretch), 1227 (C-O stretch), 1595, 1499, 1472 (aromatic C=C ring stretch), 1249 (asymmetric C-O-C stretch), 1042 (symmetric C-O-C stretch), $1648(\mathrm{C}=\mathrm{N}$ stretch), 2950 (C-H stretch $\gamma$ as $\left.\mathrm{CH}_{3}\right), 2835$ (C-H stretch $\gamma$ sy $\mathrm{CH}_{3}$ ), 1224 (C-O stretch); ${ }^{1} \mathrm{H}$ NMR (DMSO-d 6 , $\delta$ ppm): 6.87-7.18 (4H, m, aromatic protons), $3.85(3 \mathrm{H}$, $\left.\mathrm{s}, \mathrm{OCH}_{3}\right), 7.23-7.48(4 \mathrm{H}, \mathrm{m}$, aromatic protons), 5.57 $(1 \mathrm{H}, \mathrm{s}, \mathrm{Ar}-\mathrm{OH})$.

4-[5-(3,4,5-trimethoxyphenyl)-1,3,4-oxadiazol2-yl]phenol (3k). Yield: 88.82\% (2.90 g); mp: 
$244^{\circ} \mathrm{C}$; $\mathrm{R}_{\mathrm{f}}$ value: 0.813 ; UV ( $\left.\lambda_{\max }, \mathrm{nm}\right): 302.2$; IR $\left(\mathrm{KBr}, \mathrm{cm}^{-1}\right): 3060,3039$ (aromatic C-H stretch), 3591 (O-H stretch), 1598, 1493, 1455 (aromatic $\mathrm{C}=\mathrm{C}$ ring stretch), 1236 (asymmetric C-O-C stretch), 1038 (symmetric C-O-C stretch), $1636(\mathrm{C}=\mathrm{N}$ stretch), 2954 (C-H stretch $\gamma$ as $\left.\mathrm{CH}_{3}\right), 2840(\mathrm{C}-\mathrm{H}$ stretch $\gamma \mathrm{s}$ $\mathrm{CH}_{3}$ ), 1219 (C-O stretch), 830 (out-of-plane aromatic $\mathrm{C}-\mathrm{H}$ bend); ${ }^{1} \mathrm{H}$ NMR (DMSO- $\left.\mathrm{d}_{6}, \delta \mathrm{ppm}\right)$ : 6.37-6.87 $(4 \mathrm{H}, \mathrm{m}$, aromatic protons), 6.92-7.21 $(2 \mathrm{H}$, $\mathrm{m}$, aromatic protons), $3.86\left(9 \mathrm{H}, \mathrm{s}, \mathrm{OCH}_{3}\right), 5.56(1 \mathrm{H}$, $\mathrm{s}, \mathrm{OH})$.

4-(5-pyridinyl-3-yl-1,3,4-oxadiazol-2-yl)phenol (3l). Yield: $64.85 \%(1.55 \mathrm{~g})$; $\mathrm{mp}: 254^{\circ} \mathrm{C}$; $\mathrm{R}_{\mathrm{f}}$ value: 0.782; UV $\left(\lambda_{\max }, \mathrm{nm}\right): 279.0$; IR $\left(\mathrm{KBr}, \mathrm{cm}^{-1}\right): 3080$ (aromatic C-H stretch), 3605 (O-H stretch), 1222 (C$\mathrm{O}$ stretch), 1604, 1499, 1472 (aromatic $\mathrm{C}=\mathrm{C}$ ring stretch), 1252 (asymmetric C-O-C stretch), 1049 (symmetric C-O-C stretch), 1647 ( $\mathrm{C}=\mathrm{N}$ stretch), 750 (out-of-plane aromatic $\mathrm{C}-\mathrm{H}$ bend); ${ }^{1} \mathrm{H}$ NMR (DMSO$\left.\mathrm{d}_{6}, \delta \mathrm{ppm}\right): 6.92-7.07(4 \mathrm{H}, \mathrm{m}$, aromatic protons), 7.11-7.42 $(4 \mathrm{H}, \mathrm{m}$, aromatic protons $), 5.55(1 \mathrm{H}, \mathrm{s}$, $\mathrm{OH})$.

4-[5-(4-hydroxyphenyl)-1,3,4-oxadiazol-2-

yl]benzene-1,3-diol (3m). Yield: 75.85\% (2.050 g); mp: $292^{\circ} \mathrm{C}$; $\mathrm{R}_{\mathrm{f}}$ value: 0.826 ; UV ( $\left.\lambda_{\max }, \mathrm{nm}\right)$ : 277.0 ; IR $\left(\mathrm{KBr}, \mathrm{cm}^{-1}\right): 3046$ (aromatic C-H stretch), 3596 (O-H stretch), 1224 (C-O stretch), 1598, 1497, 1473 (aromatic $\mathrm{C}=\mathrm{C}$ ring stretch), 1249 (asymmetric C-OC stretch), 1036 (symmetric C-O-C stretch), 1635 $(\mathrm{C}=\mathrm{N}$ stretch $) ;{ }^{1} \mathrm{H}$ NMR $\left(\mathrm{DMSO}-\mathrm{d}_{6}, \delta \mathrm{ppm}\right)$ : 6.92$7.03(4 \mathrm{H}, \mathrm{m}$, aromatic protons), 7.27-7.32 $(3 \mathrm{H}, \mathrm{m}$, aromatic protons), $5.59(3 \mathrm{H}, \mathrm{s}, \mathrm{OH})$.

\section{4-[5-(3-aminophenyl)-1,3,4-oxadiazol-2-}

yl]phenol (3n). Yield: $70.28 \%(1.78 \mathrm{~g})$; $\mathrm{mp}: 210^{\circ} \mathrm{C}$; $\mathrm{R}_{\mathrm{f}}$ value: 0.698 ; $\mathrm{UV}\left(\lambda_{\max }, \mathrm{nm}\right)$ : 286.0 ; IR $\left(\mathrm{KBr}, \mathrm{cm}^{-}\right.$ $\left.{ }^{1}\right)$ : 3602 (O-H stretch), 3042 (aromatic C-H stretch), 1230 (C-O stretch), 1604, 1490, 1462 (aromatic $\mathrm{C}=\mathrm{C}$ ring stretch), 1252 (asymmetric $\mathrm{C}-\mathrm{O}-\mathrm{C}$ ring stretch), 1039 (symmetric C-O-C stretch), 1646 (C=N stretch), 3515 (N-H stretch for aromatic primary amine, asymmetric), 3421 (N-H stretch, primary amine, symmetric), 1290 (C-N stretch for aromatic primary amine); ${ }^{1} \mathrm{H}$ NMR (DMSO-d ${ }_{6}, \delta$ ppm): 6.92-7.13 (4H, $\mathrm{m}$, aromatic protons), 7.13-7.4 $(4 \mathrm{H}, \mathrm{m}$, aromatic protons), $4.40\left(2 \mathrm{H}, \mathrm{s}, \mathrm{NH}_{2}\right), 5.60(1 \mathrm{H}, \mathrm{s}, \mathrm{OH})$.

\section{2-[5-(4-hydroxyphenyl)-1,3,4-oxadiazol-2-yl]-}

6-methylphenol (3o). Yield: 68.96\% (1.85 g); mp: $221^{\circ} \mathrm{C}$; $\mathrm{R}_{\mathrm{f}}$ value: 0.782 ; UV ( $\left.\lambda_{\max }, \mathrm{nm}\right)$ : 282.0 ; IR $\left(\mathrm{KBr}, \mathrm{cm}^{-1}\right)$ : 3045 (aromatic C-H stretch), $3599(\mathrm{O}-\mathrm{H}$ stretch), 1228 (C-O stretch), 1604, 1491, 1462 (aromatic $\mathrm{C}=\mathrm{C}$ ring stretch), 1246 (asymmetric $\mathrm{C}-\mathrm{O}$ $\mathrm{C}$ stretch), 1038 (symmetric C-O-C stretch), 1654 (C=N stretch), 2962 (methyl C-H stretch $\gamma$ as $\mathrm{CH}_{3}$ ), 2872 (methyl C-H stretch $\gamma \mathrm{s} \mathrm{CH}_{3}$ ), 1450 (C-H bend $\delta$ as $\left.\mathrm{CH}_{3}\right), 1378\left(\mathrm{C}-\mathrm{H}\right.$ bend $\left.\delta \mathrm{s} \mathrm{CH}_{3}\right), 3342(\mathrm{~N}-\mathrm{H}$ stretch, secondary amide); ${ }^{1} \mathrm{H}$ NMR (DMSO- $\mathrm{d}_{6}, \delta$ ppm): 6.67-7.03 (3H, m, aromatic protons), 7.11-7.38 $(4 \mathrm{H}, \mathrm{m}$, aromatic protons), $5.59(2 \mathrm{H}, \mathrm{s}, \mathrm{Ar}-\mathrm{OH}), 2.38$ $\left(3 \mathrm{H}, \mathrm{s}, \mathrm{Ar}-\mathrm{CH}_{3}\right)$.

Screening for Antimicrobial activity. The antimicrobial activity of all the newly synthesized compounds (3a-o) was determined by well plate method $^{13}$ in nutrient agar (Hi-Media) (antibacterial activity) and Sabouraud dextrose agar (SDA) (HiMedia) (antifungal activity). The in vitro antimicrobial activity was carried out against $24 \mathrm{~h}$ old cultures of bacterial and $72 \mathrm{~h}$ old cultures of fungal strain. The bacterial and fungal strains for the study are listed in Tables 2 and 3.

Pure cultures of the test microorganisms were procured from Universal supplies, Coimbatore, Tamilnadu. The compounds were tested at a concentration of $100 \mu \mathrm{g} / \mathrm{ml}$ and solutions were prepared were prepared in dimethyl formamide (DMF). The petridishes used for antibacterial screening were incubated at $37 \pm 1^{\circ} \mathrm{C}$ for $24 \mathrm{~h}$, while those used for antifungal activity were incubated at $28^{\circ} \mathrm{C}$ for $48-72 \mathrm{~h}$. The results were compared to Chloramphenicol $(50 \mu \mathrm{g} / \mathrm{ml})$ and Griseofulvin $(50 \mu \mathrm{g} / \mathrm{ml})$ for antibacterial and antifungal activity respectively by measuring zone of inhibition in $\mathrm{mm}$. The antibacterial and antifungal screening results were presented in Table 2 and Table 3. 
Table 2. Antibacterial screening results of 2,5-disubstituted-1,3,4-oxadiazoles (3a-o).

\begin{tabular}{|c|c|c|c|c|c|c|}
\hline \multirow[b]{3}{*}{ Compound } & \multicolumn{6}{|c|}{ Antibacterial activity } \\
\hline & \multicolumn{6}{|c|}{ Zone of inhibition (mm) } \\
\hline & $\begin{array}{c}\text { Staphylococcus } \\
\text { aureus } \\
\text { (ATCC 25923) }\end{array}$ & $\begin{array}{c}\text { Bacillus subtilis } \\
\text { (ATCC 6633) }\end{array}$ & $\begin{array}{c}\text { Bacillus } \\
\text { megaterium } \\
\text { (ATCC 1327) }\end{array}$ & $\begin{array}{c}\text { Escherichia coli } \\
\text { (ATCC 25922) }\end{array}$ & $\begin{array}{c}\text { Pseudomonas } \\
\text { aeruginosa } \\
\text { (ATCC 27853) }\end{array}$ & $\begin{array}{c}\text { Shigella } \\
\text { dysenteriae } \\
\text { (ATCC 13313) }\end{array}$ \\
\hline $3 a$ & 14 & 13 & 13 & 10 & 15 & 12 \\
\hline $3 b$ & 18 & 21 & 17 & 21 & 17 & 15 \\
\hline $3 c$ & 14 & 13 & 15 & 13 & 12 & 14 \\
\hline $3 d$ & 15 & 17 & 16 & 20 & 15 & 14 \\
\hline $3 \mathrm{e}$ & 19 & 24 & 20 & 22 & 18 & 15 \\
\hline $3 f$ & 15 & 16 & 16 & 16 & 14 & 12 \\
\hline $3 g$ & 19 & 23 & 21 & 23 & 18 & 16 \\
\hline $3 h$ & 17 & 21 & 19 & 21 & 17 & 15 \\
\hline $3 \mathrm{i}$ & 15 & 17 & 16 & 17 & 14 & 15 \\
\hline $3 \mathrm{j}$ & 16 & 22 & 17 & 21 & 17 & 14 \\
\hline $3 \mathrm{k}$ & 14 & 16 & 14 & 18 & 14 & 15 \\
\hline 31 & 11 & 12 & 10 & 09 & 12 & 11 \\
\hline $3 \mathrm{~m}$ & 17 & 22 & 16 & 22 & 17 & 15 \\
\hline $3 n$ & 19 & 21 & 17 & 21 & 15 & 15 \\
\hline 30 & 15 & 19 & 16 & 17 & 14 & 15 \\
\hline $\begin{array}{l}\text { Chloramphenicol } \\
\quad(50 \mu \mathrm{g} / \mathrm{ml})\end{array}$ & 20 & 24 & 21 & 24 & 19 & 17 \\
\hline
\end{tabular}

Table 3. Antifungal screening results of 2,5-disubstituted-1,3,4-oxadiazoles (3a-o).

\begin{tabular}{|c|c|c|c|}
\hline \multirow{3}{*}{ Compound } & \multicolumn{3}{|c|}{ Antifungal activity } \\
\hline & \multicolumn{3}{|c|}{ Zone of inhibition (mm) } \\
\hline & $\begin{array}{l}\text { Candida albicans } \\
\text { (ATCC 10231) }\end{array}$ & $\begin{array}{l}\text { Aspergillus niger } \\
\text { (ATCC 16404) }\end{array}$ & $\begin{array}{c}\text { Aspergillus flavus } \\
\text { (ATCC 22547) }\end{array}$ \\
\hline $3 a$ & 18 & 17 & 16 \\
\hline $3 b$ & 15 & 14 & 13 \\
\hline $3 c$ & 14 & 13 & 12 \\
\hline $3 d$ & 16 & 17 & 17 \\
\hline $3 e$ & 18 & 18 & 15 \\
\hline $3 f$ & 14 & 16 & 14 \\
\hline $3 g$ & 19 & 19 & 17 \\
\hline $3 \mathrm{~h}$ & 17 & 18 & 17 \\
\hline $3 \mathrm{i}$ & 17 & 18 & 18 \\
\hline $3 \mathrm{j}$ & 15 & 14 & 16 \\
\hline $3 \mathrm{k}$ & 16 & 17 & 16 \\
\hline 31 & 12 & 12 & 13 \\
\hline $3 \mathrm{~m}$ & 15 & 14 & 13 \\
\hline $3 n$ & 17 & 17 & 16 \\
\hline 30 & 15 & 14 & 14 \\
\hline $\begin{array}{l}\text { Griseofulvin } \\
(50 \mu \mathrm{g} / \mathrm{ml})\end{array}$ & 23 & 21 & 19 \\
\hline
\end{tabular}

\section{RESULTS AND DISCUSSION}

The results of antimicrobial activity indicated that compound $\mathbf{3 b}, \mathbf{3 e}, \mathbf{3 g}, \mathbf{3 h}, \mathbf{3} \mathbf{j}, \mathbf{3 m}$ and $\mathbf{3 n}$ were found to be active against Bacillus subtilis, Staphylococcus aureus and Bacillus megaterium whereas compound $\mathbf{3 b}, \mathbf{3 d}, \mathbf{3 e}, \mathbf{3 g}, \mathbf{3 h}, \mathbf{3} \mathbf{j}$ and $\mathbf{3 m}$ exhibit significant antibacterial activity against Escherichia coli and Pseudomonas aeruginosa and Shigella dysenteriae at $100 \mu \mathrm{g} / \mathrm{ml}$ concentration as compared to chloramphenicol $(50 \mu \mathrm{g} / \mathrm{ml})$. The screening results revealed that compounds $\mathbf{3 a}, \mathbf{3 d}, \mathbf{3 e}$, 3g, 3h, 3i, 3k and $3 \mathbf{n}$ displayed better antifungal activity against Candida albicans, Aspergillus niger and Aspergillus flavus.

The antimicrobial activity of the compounds varied upon the type and position of the substituents at 5-(4-hydroxyphenyl)-1,3,4-oxadiazole moiety. It can be concluded from the antimicrobial screening results that when 5-(4-hydroxyphenyl)-1,3,4oxadiazole were substituted with p-tolulyl, 4nitrophenyl, 4-methoxyphenyl, 4-aminophenyl, 2nitrophenyl anilinyl, 2,4-dinitrophenyl anilinyl, 2,4- 
dihydroxy phenyl and 3-aminophenyl at $2^{\text {nd }}$ position, the antimicrobial activity was altered to an appreciable extent.

\section{ACKNOWLEDGEMENTS}

The authors express their sincere thanks to the Chairman, NMR Research Centre, Indian Institute of Sciences (IIS), Bangalore and to the Head, Regional Sophisticated Instrumentation Centre (RSIC), Central Drug Research Institute (CDRI), Lucknow for providing ${ }^{1} \mathrm{H}$ NMR and elemental analysis data.

\section{REFERENCES}

1. El-Emam, A.A., Al-Deeb, O.A., Al-Omar, M. and Lehmann, J. 2004. Synthesis, antimicrobial and anti-HIV activity of certain 5-(1-adamantyl)-2-substituted thio-1,3,4-oxadiazoles and5-(1-adamantyl)-3-substitutedaminomethyl-1,3,4oxadiazolin-2-thiones. Bioorg. Med. Chem. 12, 5107-5113.

2. Kucukguzel, S.G., Oruc, E.E., Rollas, S., Sahin, F. and Ozbek, A. 2002. Synthesis, characterization and biological activity of novel 4-thiazolidinones, 1,3,4-oxadiazoles and some related compounds. Eur. J. Med. Chem. 37(3), 197-206.

3. Preethi R Kagthara, Niraj S Shah, Rajeev K Doshi and Parekh, H.H. 1999. Synthesis of 2,5-disubstituted-1,3,4oxadiazoles as biologically active heterocycles. Indian $J$. Chem. 38B, 572-576.

4. Santagati, M., Modica, M., Santagati, A., Russo, F., Caruso, A., Cutuli, V., Dipietro, E. and Amico Roxas, M. 1994. Synthesis and pharmacological properties of benzothiazole, 1,3,4-oxadiazole and 1,2,4-thiadiazole derivatives. Pharmazie. 49, 880-884.
5. Unangast, P.C., Shrum, G.P., Conner, D.T., Dyer, C.D. and Schrier, D.J. 1992. Novel 1,3,4-oxadiazoles and 1,2,4thiadiazoles as dual 5-lipooxygenase and cyclooxygenase inhibitors. J. Med. Chem. 35, 3691-3698.

6. Khan, M.S.Y., Khan, R.M. and Susma Drabu. 2001. Anticonvulsant and antibacterial activity of some new 1,3,4oxadiazole derivatives. Indian J. Heterocycl. Chem. 11, 119-122.

7. Evangelia D. Chrysiha, Magda N. Kosmopoulou, Constantinos Tiraidis, Rozina Kardakaris, Nicolas Bischler, Demetres D. Leonidas, Zsuzsa Hadady, Laszlo Somsak, Tibor Dosca, Pal Gergely and Nikos G. Oikanomakos. 2005. Kinetic and crystallographic studies on 2-( $\beta$-Dglucopyranosyl)-5-methyl-1,3,4-oxadiazole, -benzthiazole, and-benzimidazole, inhibitors of muscle glycogen phosphorylase. b. Evidence for a new binding site. Protein science. 14, 873-878.

8. Maslat, A.O., Abussaud, M., Tashtoush, H. and Al-Talib, M. 2002. Synthesis, antibacterial, antifungal and genotoxic activity of bis-1,3,4-oxadiazole derivatives. Pol. J. Pharmacol. 54, 55-59.

9. Farghaly, A.A., Bekhit, A.A. and Park, J.Y. 2000. Design and synthesis of some oxadiazolyl, thiazolidinyl and thiazolyl derivatives of 1H-pyrazole as anti-inflammatory, antimicrobial agents. Arch Pharm (Weinheim). 333, 53-57.

10. Phone Poulene. 1974. FP 2202873. Chem. Abstr. 82, 111782.

11. Song Cao, Xu Hong Qian, Gonghua Song, Bing Chai and Zhisheng Jiang. 2003. Synthesis and antifeedant activity of new oxadiazolyl 3(2H)-pyridazinones. J. Agric. Food. Chem. 15, 152-155.

12. Surendra N. Pandeya. 2001. A Text book of Medicinal Chemistry, SG Publisher, Varanasi, p. 353.

13. Barry, A. 1991. Antibiotics in laboratory medicine. Williams and Wilkins, Baltimore, p. 14. 